\title{
金属配合物/手性磷酸参与的不对称接力催化反应
}

\author{
吴祥李明丽龚流柱* \\ (合肥微尺度物质科学国家实验室 中国科学技术大学 合肥 230026)
}

\begin{abstract}
摘要 金属配合物和有机小分子双体系联合催化的不对称接力催化反应是一新兴热点研究领域. 接力催化可以由易得 的起始原料生成结构复杂的化合物, 实现单一催化剂无法实现的新反应, 并且避免了对不稳定中间体的分离纯化, 提 高了反应的步骤经济性和原子经济性. 手性磷酸是近年来研究最为热门的有机小分子催化剂之一, 其与金属催化剂结 合催化也得到了深入的研究. 本工作即针对金属催化剂和手性磷酸结合的接力催化反应的研究进展, 做一简要评述. 关键词 金属配合物; 手性磷酸; 不对称; 接力催化
\end{abstract}

\section{Asymmetric Relay Catalysis Reaction Consisting of Metal Complex and Chiral Phosphoric Acids}

\author{
Wu, Xiang Li, Mingli Gong, Liuzhu* \\ (Hefei National Laboratory for Physical Sciences at the Microscale and Department of Chemistry, University of Science and \\ Technology of China, Hefei 230026)
}

\begin{abstract}
Metal complex and organo combined catalysis is a new and exciting research area and has attracted close attention. Relay catalysis (or sequential catalysis), in which both the organocatalyst and the metal catalyst perform two distinct catalytic cycles for the consecutive reactions, enables the assembling readily available starting materials into structurally complex molecules. Besides, relay catalysis can also accomplish unprecedented reactions, which are not accessible through the use of either of the catalytic systems alone. More essentially, it avoids the purification of unstable intermediate and improves process economy and atom economy. Chiral phosphoric acids become the hottest kind of organocatalysts in recent decades. Chiral phosphoric acid has been applied to asymmetric transfer hydrogenation of imines, enantioselective Friedel-Crafts reaction, Mannich reaction, hydrophosphonylation reaction, Strecker reaction, aza-Diels-Alder reaction, alkylation of diazoester reaction and so on. Chiral phosphoric acid can act as both proton donor and proton acceptor simultaneously, and the system of which combining with metal has attracted intensive study. Transition metal catalysis was dominated in catalytic organic synthesis. Owing to the explosive development of organocatalysis in recent years, the combination of transition metal catalysts and organocatalysts has displayed as a new and powerful strategy for developing new and useful reactions, and has attracted increasing attention. In this review, we summarize recent progress in the field of metal complex/phosphoric acids relay catalysis. And in our opinion, the study of new catalysis systems will continue to be an exciting research area, the inquiry of the applications in the total synthesis of natural products is worthy of further investigation and the exploration of appropriate catalysis systems applied in industrial production represents the future research direction.

Keywords metal complex; chiral phosphoric acids; asymmetric; relay catalysis
\end{abstract}

\section{1 引言}

不对称催化的方法学研究是有机化学研究的一个 重要热点领域. 进入新世纪以来, 有机小分子催化作为 不对称催化的新领域, 得到了蓬勃发展, 成为了继酶催 化和金属催化后新的广泛使用的不对称合成途径. 近年 来, 金属催化剂和有机小分子催化剂结合的多催化体 系, 成为一种新兴的研究策略和方向, 引起了化学家的 密切关注 ${ }^{[1]}$.

金属催化剂和有机小分子催化剂结合的多体系催 化反应，可以分为协同催化反应(Cooperative Catalysis or Dual catalysis)和接力催化反应(Relay Catalysis, Cascade catalysis or Sequential Catalysis)(图 1) ${ }^{[2]}$. 协同催化 反应过程中, 两种催化剂分别作用于不同底物, 活化的 两种中间体发生反应得到最终产物. 在接力催化反应过 程中，一种催化剂催化发生第一步反应，得到的产物在 另一种催化剂的作用下发生第二步反应，得到目标产 物. 接力催化反应在同一个反应容器中进行, 将多步反 应缩短为一步，避免对不稳定中间体的分离纯化，同时 可以有效地减少溶剂的消耗和后处理废弃物的排放, 极 大地提高反应的步骤经济性和原子经济性. 更重要的 是, 接力催化可以实现单一催化剂无法实现的反应 ${ }^{[3]}$.

\footnotetext{
*E-mail: gonglz@ustc.edu.cn; Tel.: 0086-0551-63600671; Fax: 0086-0551-63606266

Received March 14, 2013; published April 17, 2013.

Project supported by the Fundamental Research Funds for the Central Universities (No. WK2340000043).

项目受中央高校基本科研业务费专项资金(No. WK2340000043)资助.
} 


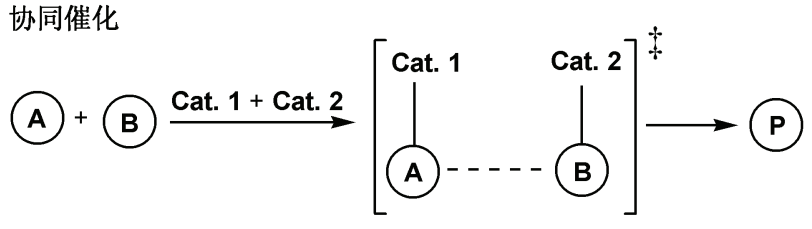

接力催化

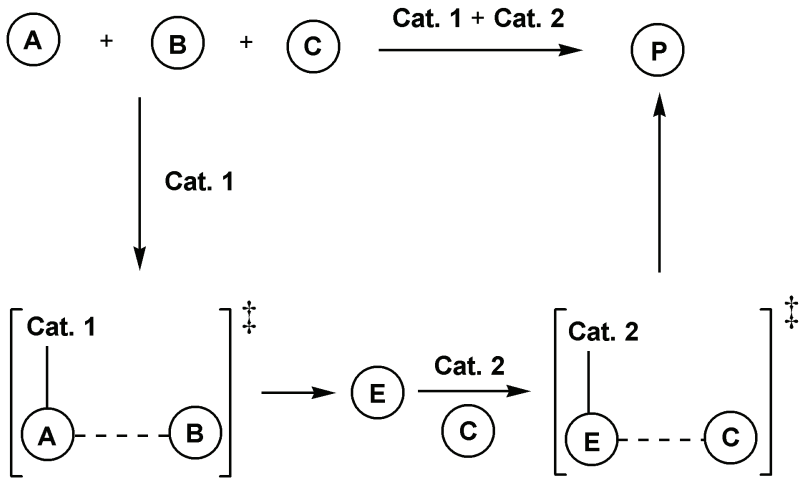

图 1 协同催化和接力催化

Figure 1 Cooperative catalysis and relay catalysis

手性磷酸是近年来研究最为热门的有机小分子催 化剂之一 ${ }^{[4]}$, 其与金属催化剂结合催化也得到了深入的 研究. 与其它有机手性布朗斯特酸催化剂相比, 手性磷 酸具有以下主要特征(图 2): (1)手性磷酸具有较强酸性, 与底物形成离子对或氢键从而活化底物; (2)磷氧双键上 的氧可以作为路易斯碱活化亲电试剂, 因此手性磷酸是 一种双功能催化剂; (3)手性联二荎酚骨架的 3,3'-位引入 不同的取代基, 可以对催化剂的空间结构进行微调以控 制反应的立体选择性. 手性磷酸由于其特殊的结构和优 异的催化性能, 在各类有机小分子催化剂中脱颖而出, 得到了研究者的广泛关注.

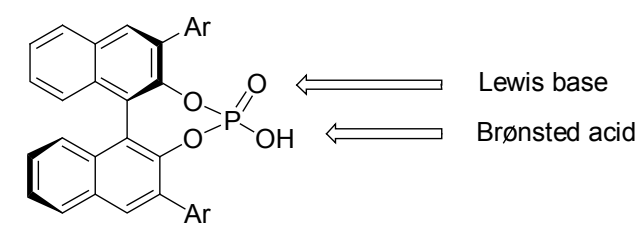

图 2 手性磷酸的双功能特性

Figure 2 Bifunctional character of chiral phosphoric acids

近年来很多综述性论文已对过渡金属催化剂和有 机小分子催化剂结合的多催化体系进行了专门的评述. 本文在综合前述文献的基础上, 针对金属催化剂和手性 磷酸结合的接力催化反应的研究进展, 进行简要评述.

\section{2 钉配合物/磷酸体系}

2008 年, Terada 课题组 ${ }^{[5]}$ 首次报道了钉催化剂与非 手性磷酸接力催化的烯烃异构化/Friedel-Crafts 反应(图 3). 在反应中, 烯丙基酰胺在 $1 \mathrm{~mol} \%\left[\mathrm{RuH}(\mathrm{CO})\left(\mathrm{PPh}_{3}\right)_{3}\right]$ 的催化作用下, 异构为烯胺, 进而在非手性磷酸催化作
用下异构为亚胺，再在该磷酸的催化下进一步与富电子 芳香化合物发生 Friedel-Crafts 反应. 作者未能对该反应 实现不对称催化.
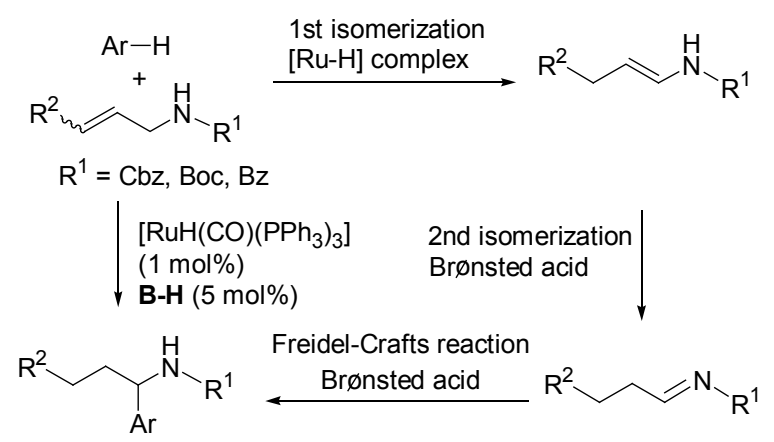

up to $98 \%$ yield

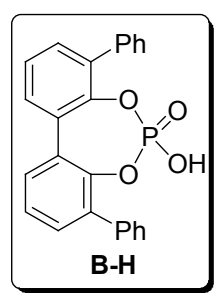

图 3 烯烃异构化/Friedel-Crafts 串联反应

Figure 3 Alkenes isomerization/Friedel-Crafts cascade reaction

游书力课题组 ${ }^{[6]}$ 将烯烃复分解催化和手性磷酸催化 结合起来, 发展了烯烃复分解/分子内 Friedel-Crafts 串 联反应，以最高 $97 \%$ 的收率和最高 $94 \%$ 的 $e e$ 值得到多环 吲哚衍生物(图 4). 在 $5 \mathrm{~mol} \%$ 钓催化剂(Zhan-1B)作用下, 2 - 吲哚基烯和 $\alpha, \beta$-不饱和酮发生烯烃复分解生成
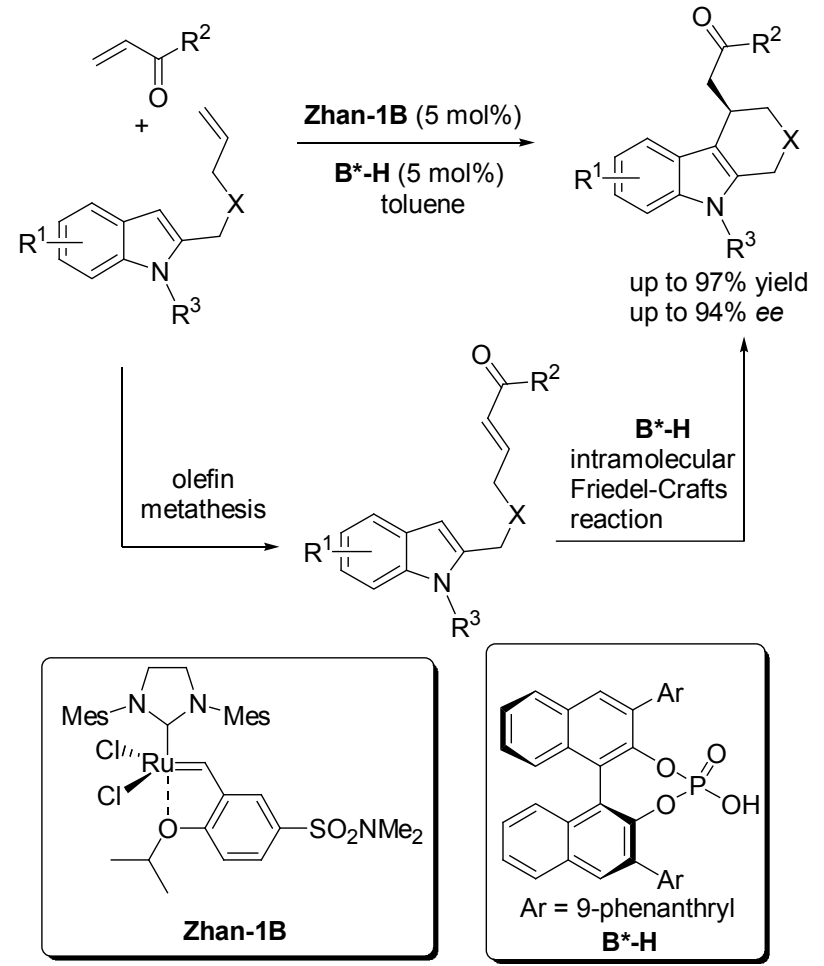

图 4 烯烃复分解/分子内 Friedel-Crafts 串联反应

Figure 4 Olefin metathesis/intramolecular Friedel-Crafts cascade reaction 
吲哚基烯酮, 然后在手性磷酸催化下发生分子内 Friedel-Crafts 反应. 事实上，反应中所用的烯烃复分解 催化剂本身具有一定的路易斯酸性, 也可以催化第二步 分子内 Friedel-Crafts 反应 ${ }^{[7]}$, 而手性磷酸具有更高的催 化活性，因此能高对映选择性地得到目标产物.

随后, 游书力课题组 ${ }^{[8]}$ 利用相似的方法, 发展了烯 烃复分解/分子内氮杂 Michael 加成串联反应. 在酸性条 件下, 首次实现了没有拉电子基团取代的吲哚环上氮原 子的不对称烷基化反应(图 5).

最近, 游书力课题组 ${ }^{[9]}$ 再次利用钉金属化合物 (Hoveyda-Grubbs II 催化剂)和手性磷酸组成的双体系催 化, 完成了烯烃复分解/异构化/分子内 Pictet-Spengler三 步串联反应, 合成得到四氢- $\beta$-咔巴啉类衍生物, 收率达
到 98\%,ee 值高达 99\%(图 6).

从上述几个反应可以看到, 游书力课题组将应用广 泛的烯烃复分解催化剂与手性磷酸相结合, 高对映选择 性地合成得到含氮的多环化合物. 这些杂环化合物的骨 架在天然产物中广泛存在. 这也为不对称接力催化的发 展提供了很好的方向.

2011 年, 周永贵和㚞红军课题组 ${ }^{[10]}$ 报道了在钉配 合物和手性磷酸接力催化体系中二氢喹啞啉转化为手 性四氢喹惡啉的不对称歧化反应(图 7). 收率达到 $98 \%$, $e e$ 值达到 $94 \%$. 反应的高对映选择性归结于主反应的速 率 $k_{2}$ 明显大于钓催化的副反应速率 $k_{3}$. 该反应以氢气作 为氢源, 是一个理想的原子经济性化学转化过程.
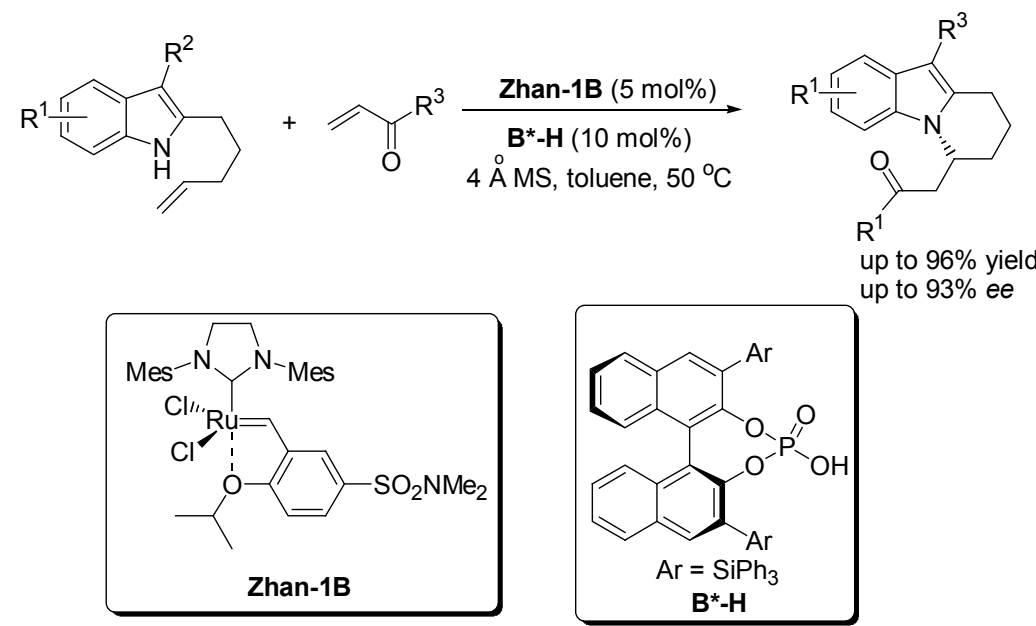
up to $93 \%$ ee

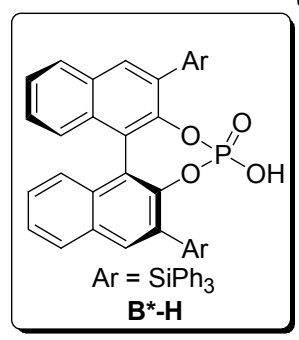

图 5 烯烃复分解/分子内 Michael 加成串联反应

Figure 5 Olefin metathesis/intramolecular Michael addition cascade reaction<smiles>[R]CC([R])N(CCc1c[nH]c2cc[R1]cc12)C(=O)C=C</smiles>

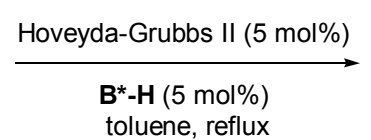<smiles>[R][R]C12CCC(=O)N1CCc1[R]3:[R]:[R]ccc3[nH]c12</smiles>

up to $98 \%$ yield<smiles>[R1]c1ccc2[nH]cc(CCN3C(=O)C=CC3[R])c2c1</smiles>
$[\mathrm{Ru}]$ up to $99 \%$ ee
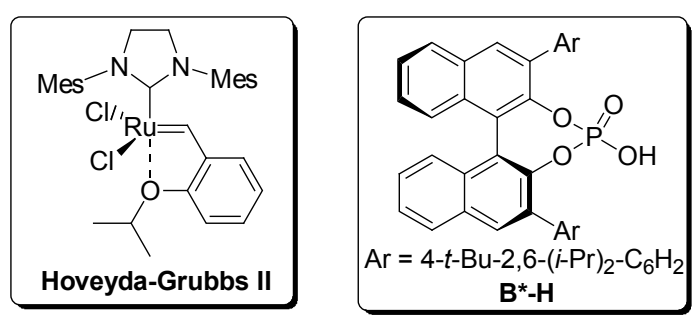

图 6 烯烃复分解/异构化/分子内 Pictet-Spengler 串联反应

Figure 6 Olefin metathesis/isomerization/intramolecular Pictet-Spengler cascade reaction 


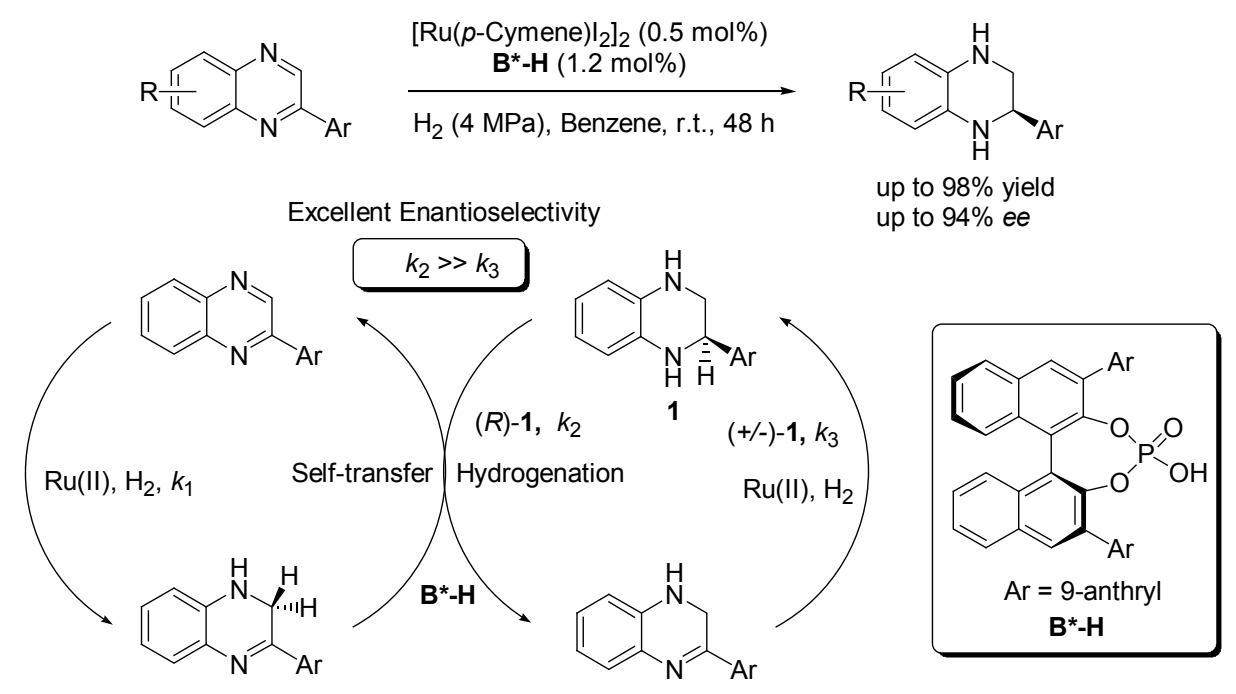

图 7 氢化/自转移氢化反应

Figure 7 Hydrogenation/self-transfer hydrogenation reaction

之后, 周永贵课题组发现利用钓配合物在氢气存在 下可以还原 Hantzsch 吡啶为 Hantzsch 酯, 实现 Hantzsch 酯的再生. 在此基础上, 作者利用钓配合物与手性磷酸 接力催化体系发展了一种苯并噁嗪酮的仿生不对称氢 化过程 ${ }^{[11]}$ (图 8), 反应可以得到高达 $99 \%$ 的 $e e$ 值. 该课 题组 ${ }^{[12]}$ 还发现二氢菲啶可以作为一种新型更易于再生 的仿 NADP 模型, 使得反应可以在更温和的条件下进 行, 从而有利于实现更多底物的高对映选择性还原.

\section{3 铑配合物/手性磷酸体系}

2012 年, Terada 课题组 ${ }^{[13]}$ 报道了铑配合物/手性磷 酸双体系催化的串联反应(图 9). 重氮化合物在铑配合 物的作用下生成崖基叶立德, 之后在手性磷酸催化下发 生对映选择性地转移氢化, 再原位用苯甲酰基保护得到 目标产物, 可以得到 $92 \%$ 的 $e e$ 值. 该反应在仅有手性铑
配合物作为催化剂时也能发生, 但所得产物没有对映选 择性，而将手性磷酸引入该体系后，手性磷酸能够通过 质子化羰基叶立德生成稳定的离子对，从而控制还原过 程的立体选择性.

\section{4 镍配合物/手性磷酸体系}

2009 年, Terada 课题组 ${ }^{[14]}$ 发展了镍氢配合物和手性 磷酸接力催化的烯烃异构化/aza-Petasis-Ferrier 反应(图 10). N,O-胺缩醛在镍氢配合物催化下, 高选择性异构化 为顺式烯醇胺缩醛. 烯醇胺缩醛在手性磷酸的催化下发 生 aza-Petasis-Ferrier 反应，原位还原，以高达 99\%的 ee 值得到手性-氨基醇类化合物. 但在该反应中过渡金属 催化的反应与手性磷酸催化的反应是在两步操作中独 立进行的, 并非严格意义上的接力催化.<smiles>[R]c1nc2ccccc2oc1=O</smiles>

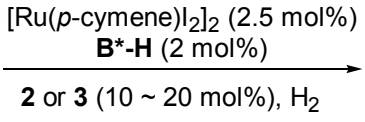

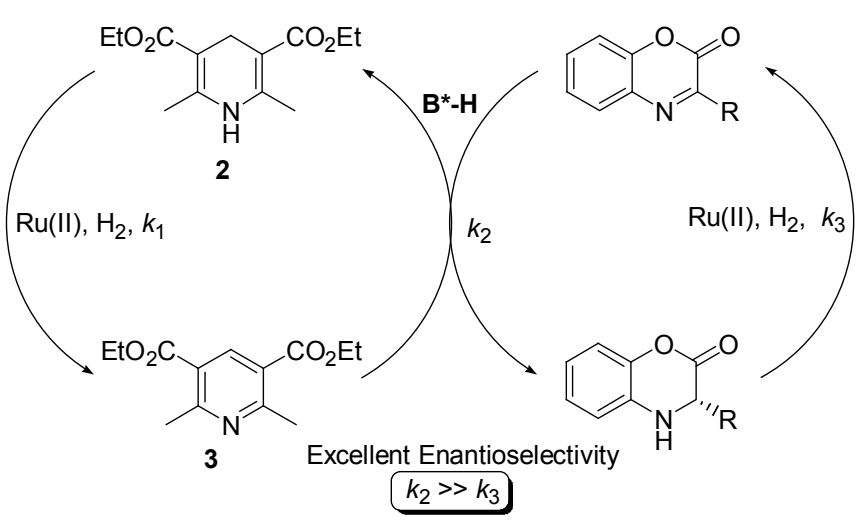<smiles>[R]C1Nc2ccccc2OC1=O</smiles>

up to $98 \%$ yield up to $99 \%$ ee

图 8 氢化/转移氢化反应

Figure 8 Hydrogenation/transfer hydrogenation reaction 


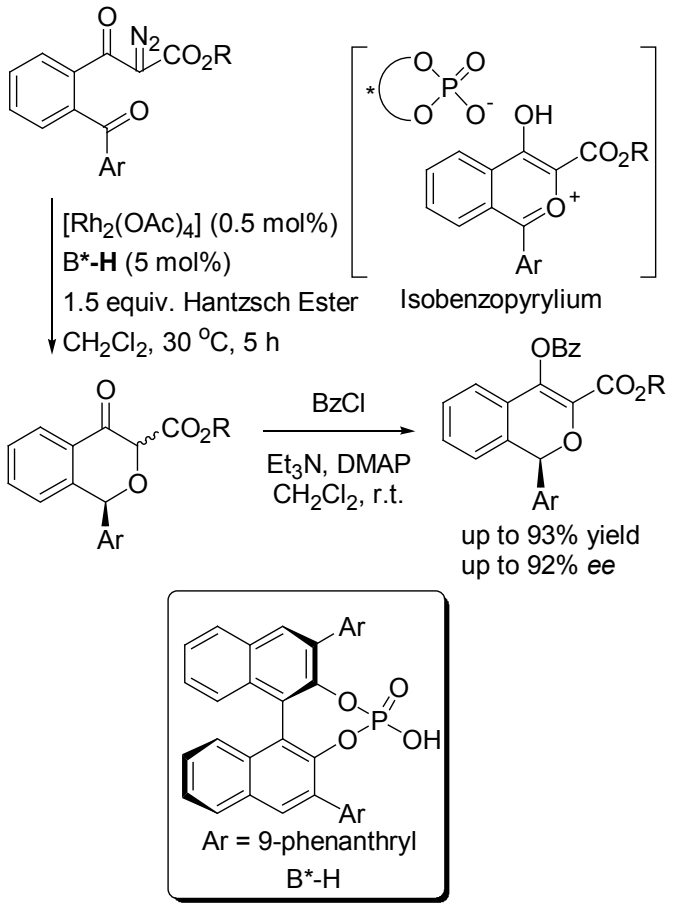

图 9 羰基叶立德生成/转移氢化反应

Figure 9 Carbonyl ylide generation/transfer hydrogenation reaction

\section{5 镁配合物/手性磷酸体系}

2012 年, 龚流柱课题组 ${ }^{[15]}$ 报道了 2-氨基苯甲醛、酮 类化合物与羰基取代的活泼亚甲基化合物在非手性路 易斯酸 $\operatorname{Mg}(\mathrm{OTf})_{2}$ 和手性磷酸的接力催化作用下, 一步 生成含有多个手性中心的四氢喹啉类衍生物, ee 值最高 达到 98\%(图 11), 该催化过程由 $\mathrm{Mg}(\mathrm{OTf})_{2}$ 或手性磷酸催 化的 Friedländer缩合反应和手性磷酸催化的转移氢化反 应组成.

\section{6 金配合物/手性磷酸体系}

近年来，金配合物在催化碳碳多重键的亲核加成反

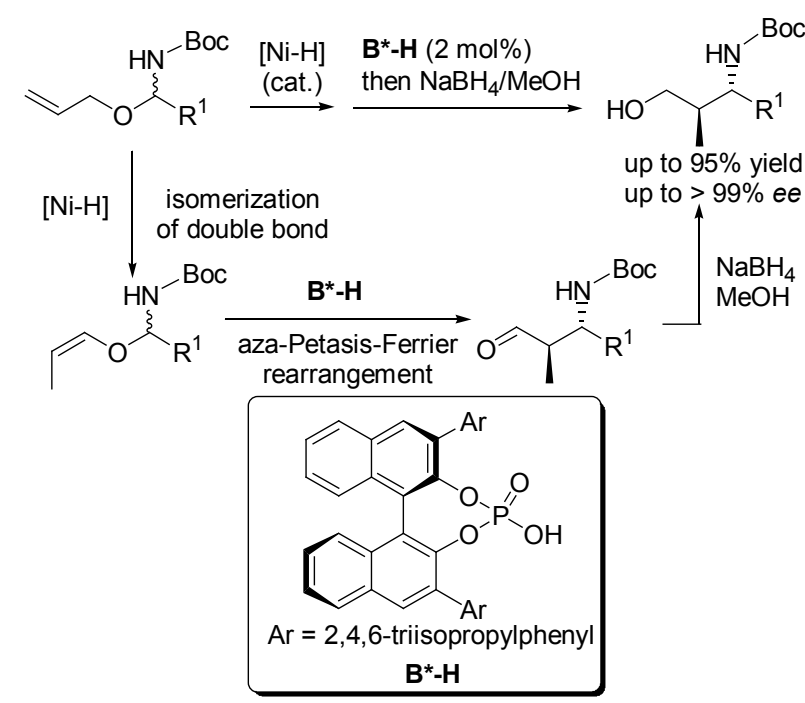

图 10 烯烃异构化/aza-Petasis-Ferrier 重排反应

Figure 10 Alkenes isomerization/aza-Petasis-Ferrier rearrangement reaction

应上表现出强大的催化活性 ${ }^{[16]}$, 如炔烃的胺化反应 ${ }^{[17]}$. 此外，金配合物也被成功应用于与手性磷酸相结合的催 化体系，显示出了良好的催化活性和兼容性.

2009 年, 龚流柱课题组 ${ }^{[18]}$ 报道了非手性金络合物 和手性磷酸接力催化的分子内氢胺化/转移氢化合成四 氢喹啉的反应. 以最高大于 $99 \%$ 的 $e e$ 值得到目标化合物 (图 12). 值得注意的是，该苯胺底物上无论是拉电子基 才还是中性或给电子基团, 都能够以很好的收率和对映 选择性得到四氢喹啉衍生物.

随后, Che 课题组 ${ }^{[19]}$ 报道了类似上述催化体系的炔 烃氢胺化/转移氢化反应, 即以炔烃和苯胺为原料, 在该 催化体系下直接转化为手性胺类化合物, 产物具有很高 的对映选择性(图 13). 接力催化的策略不仅为手性胺的 合成提供了有效的方法, 还为设计其他不对称反应提供 了重要的指导思想.

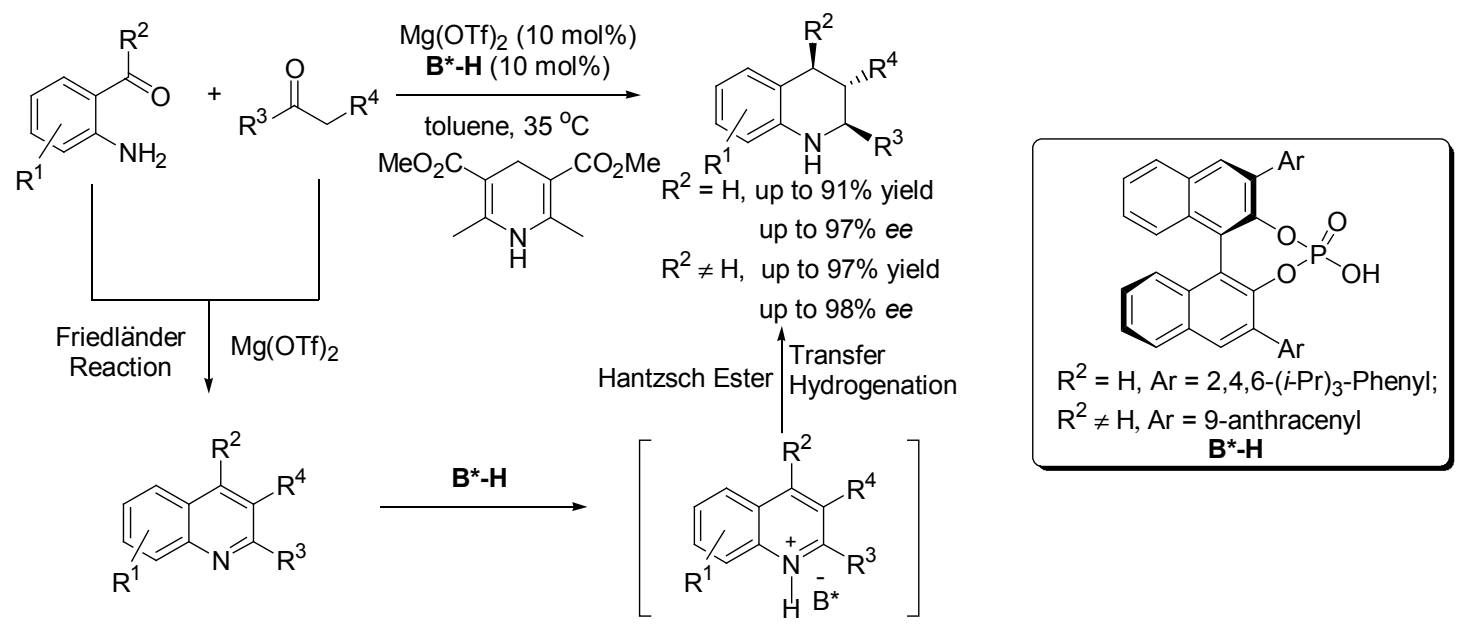

图 11 Friedländer 缩合/转移氢化反应

Figure 11 Friedländer condensation/transfer hydrogenation reaction 


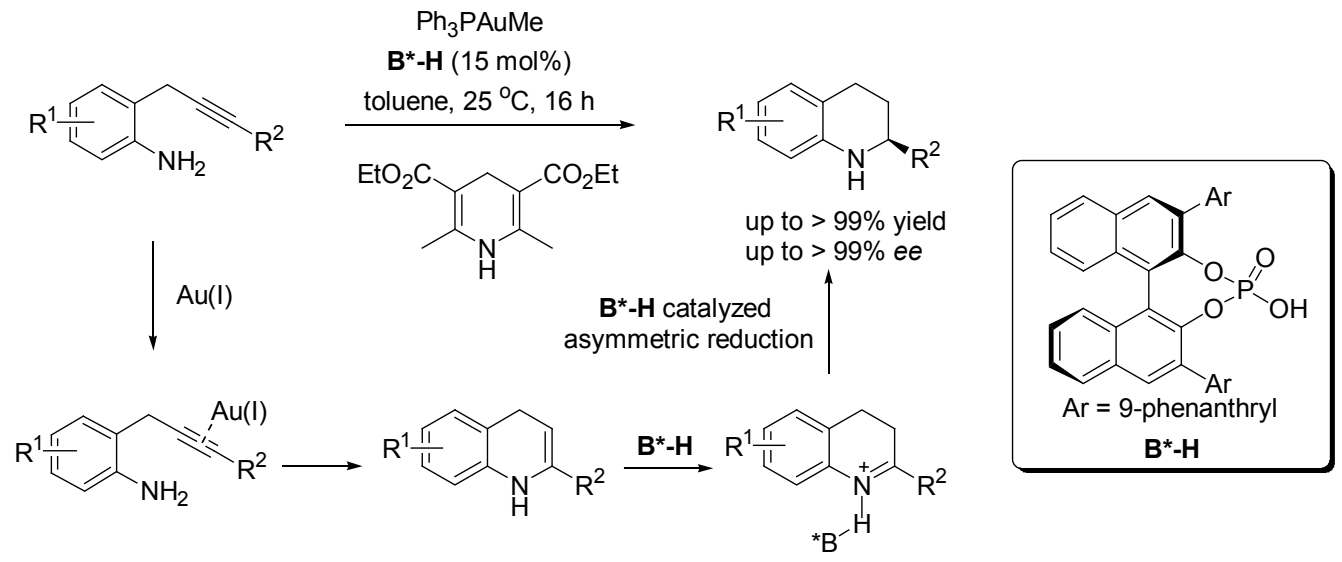

图 12 炔烃氢胺化/转移氢化反应

Figure 12 Hydroamination of alkynes/transfer hydrogenation reaction

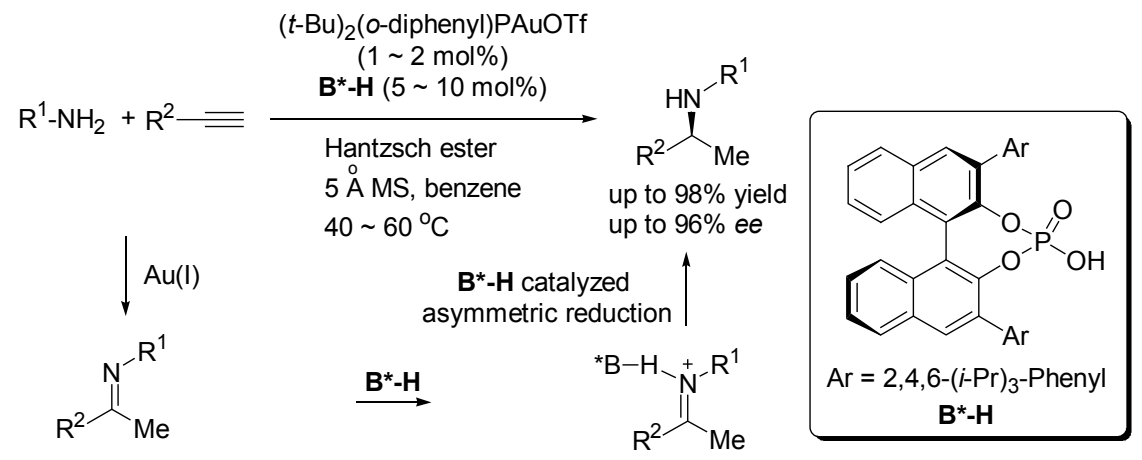

图 13 炔烃氢胺化/转移氢化反应

Figure 13 Hydroamination of alkynes/transfer hydrogenation reaction

2010 年, 龚流柱课题组 ${ }^{[20]}$ 报道了金配合物和手性 磷酸组成的催化体系参与的三组分串联不对称反应，该 串联反应由手性磷酸催化的不对称 Povarov 反应和炔烃 的氢胺化反应组成, 该反应提供了一种高对映选择性地
合成结构复杂多样的久洛里定类化合物的独特途径(图 14). 动力学研究表明, 手性磷酸不仅催化第一步不对称 Povarov 反应，而且对之后发生的金配合物催化的炔烃 氢胺化反应也起到进一步的促进作用.
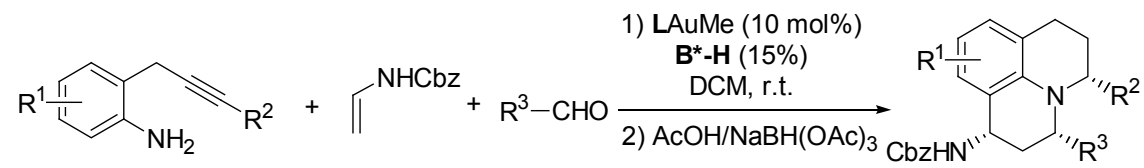

$$
\text { up to } 72 \% \text { yield }
$$

up to $>99 \%$ ee
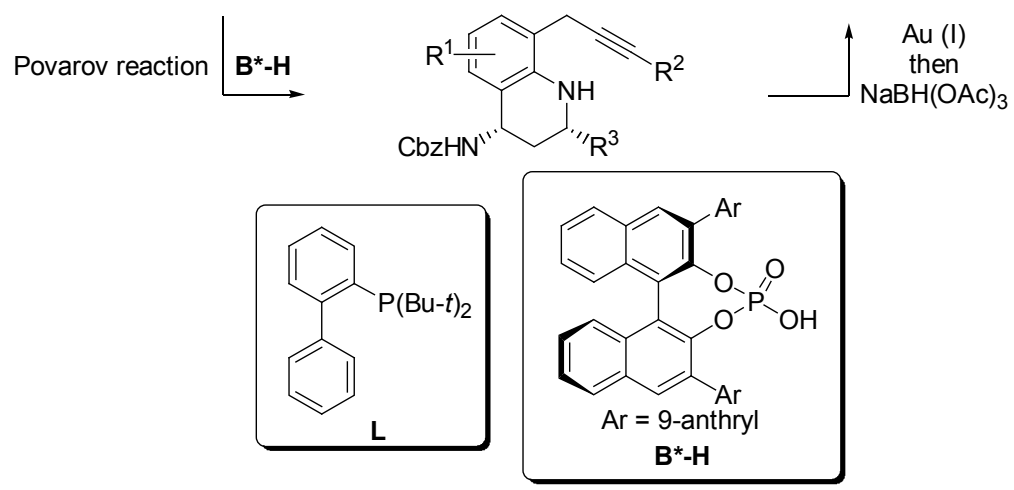

图 14 Povarov/炔烃氢胺化反应

Figure 14 Povarov/Hydroamination of alkynes reaction 
2012 年, 龚流柱课题组 ${ }^{[21]}$ 利用金/钯/磷酸三重催化 体系，实现了氢胺化/烯丙基烷基化的串联反应，高收率 地合成吡咯烷衍生物(图 15). 其中, 金催化了氢胺化, 钯和磷酸共催化了烯丙基烷基化反应. 但该篇报道中没 有不对称的相关内容.

Patil 课题组 ${ }^{[22]}$ 最近报道了利用金配合物与手性磷 酸的接力催化体系, 以 2-炔基苯甲醛和 2-氨基苯甲酰胺 为底物, 合成了具有高 $e e$ 值的 1,2-二氢异喹啉类化合物 (图 16). 其中, 金配合物活性的调节是获得高 $e e$ 值产物
的关键因素.

随后, Patil 课题组 ${ }^{[23]}$ 又报道了 2-氨基苯甲醛和端炔 在金(I)/胺/手性磷酸的催化作用下生成 2-取代四氢喹啉 的不对称接力催化反应(图 17). 控制实验表明，金(I)、 对甲氧基苯胺和手性磷酸三者同时作用于底物.

2009 年, Dixon 小组 ${ }^{[24]}$ 报道了金催化的炔基羧酸环 化与取代色胺的分子内酰亚胺化, 在手性磷酸催化下发 生的酰亚胺正离子环化接力串联反应，一步构建手性多 环吲哚衍生物(图 18), ee 值可达 96\%.

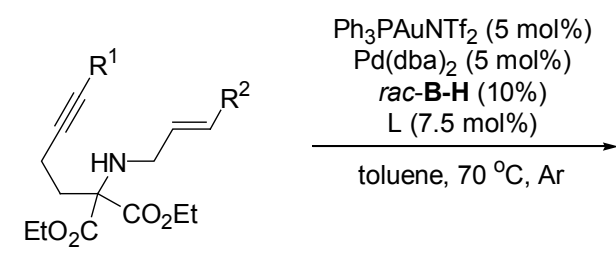

$$
\downarrow A u(l)
$$<smiles>[R]C=CCNC(CCC#CC)(OCC)C(=O)OCC</smiles><smiles>[R]C=CCC1C(=C[R])CCC1(COCC)OCC</smiles><smiles>[R]C=CCC([R])C1=NC(C(=O)OCC)(C(=O)OCC)CC1</smiles>

up to $82 \%$ yield<smiles>[R7]C(C/C=C(/[R])C)=C1CCC(COCC)(C(=O)OCC)N1[B]C</smiles>
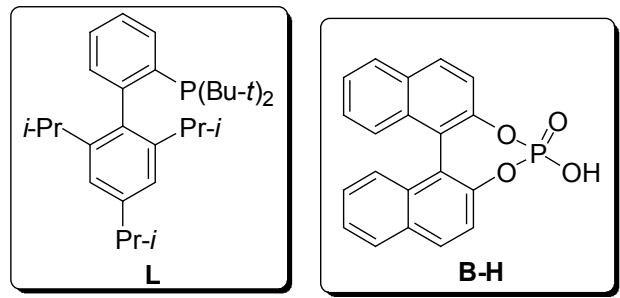

图 15 炔烃氢胺化/烯丙基烷基化串联反应

Figure 15 Hydroamination of alkynes/allylic alkylation cascade reaction
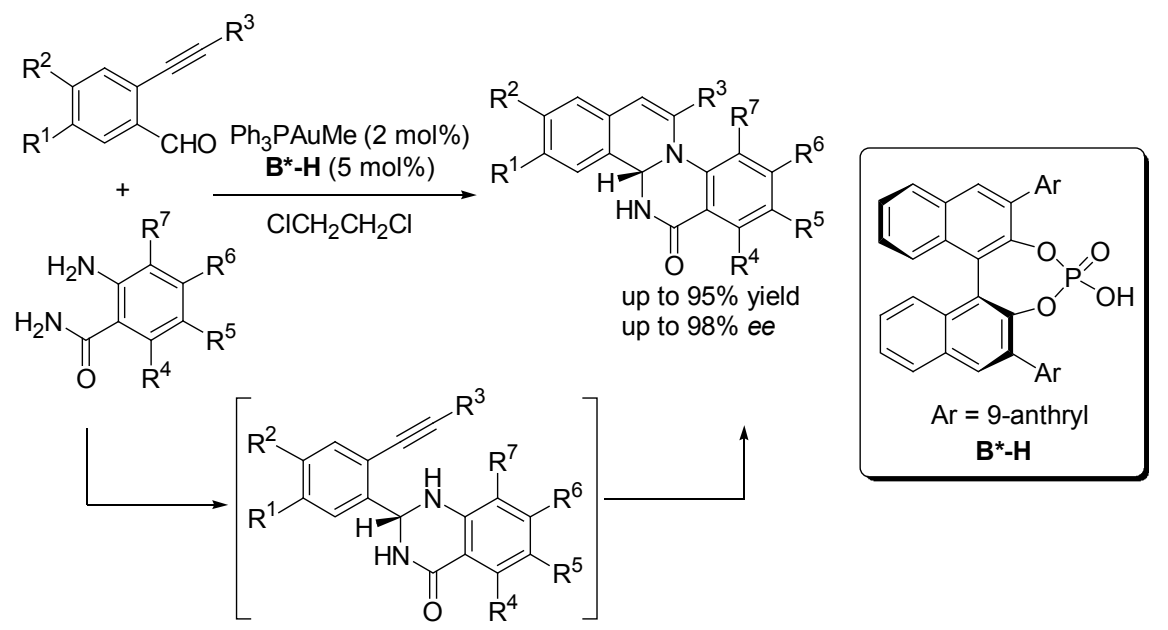

图 16 醛基缩合/炔烃氢胺化串联反应

Figure 16 Condensation/hydroamination of alkynes cascade reaction

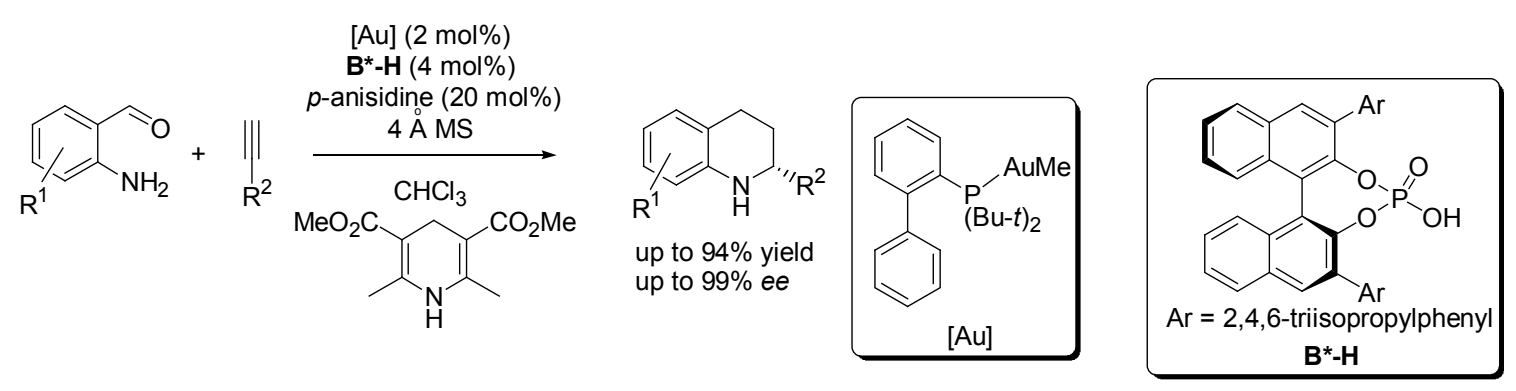

图 17 合成喹啉/转移氢化串联反应

Figure 17 Generation of quinoline/hydroamination of alkynes cascade reaction 


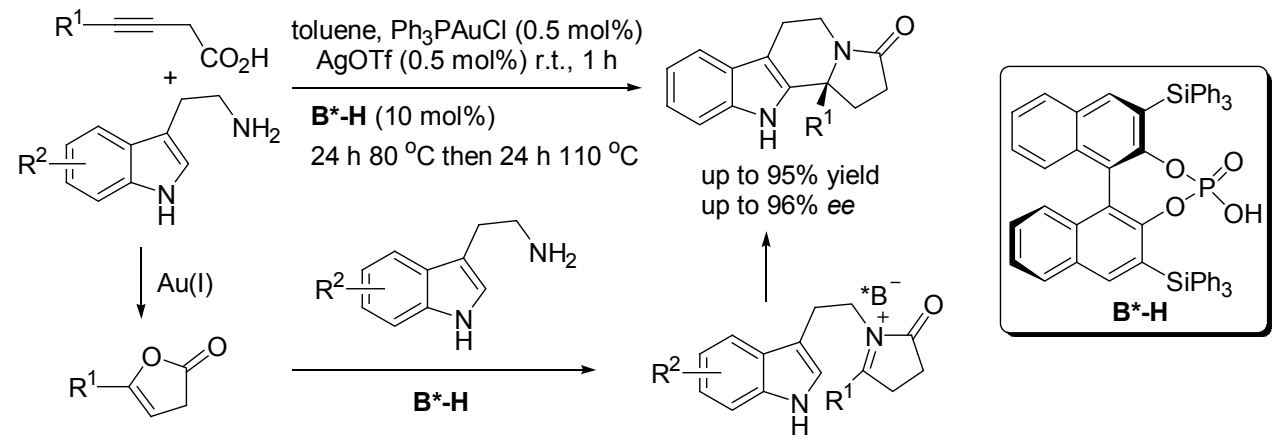

图 18 内酯化/酰亚胺正离子环化反应

Figure 18 Lactonization/ $N$-acyliminium cyclization reaction

2011 年, 龚流柱课题组 ${ }^{[25]}$ 利用金配合物和手性磷 酸组成的接力催化体系, 首次完成了炔醇参与的不对称 串联反应(图 19). 磷酸金能够用于催化呋内酯与烯醇醚 之间的反应, 并且能有效控制反应的立体选择性. 该串 联反应简单高效地合成了含有两个相邻季碳手性中心
的氨基酸衍生物，能够用于强张力多肽的合成. 但非对 映选择性还需进一步提高.

最近, 龚流柱课题组 ${ }^{[26]}$ 又报道了水杨醛、苯胺和炔 基醇三组分在金和手性磷酸的接力催化作用下生成螺 环缩醖的串联反应(图 20). 炔基醇在金的作用下发生环

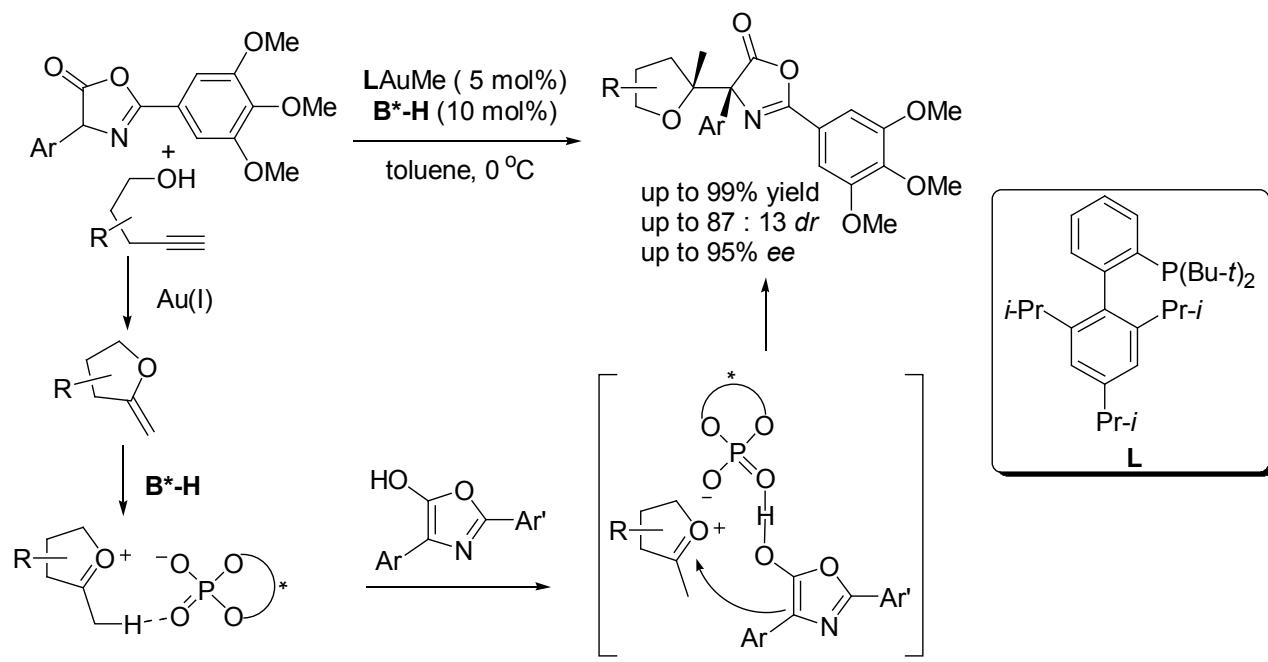

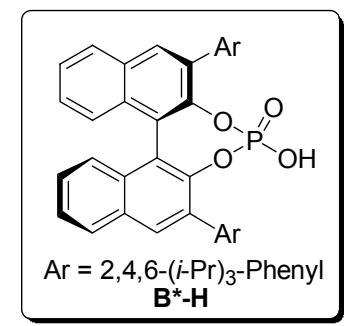

图 19 炔醇环化/呋内酯加成反应

Figure 19 Cyclization of alkynols/addition of azlactones reaction

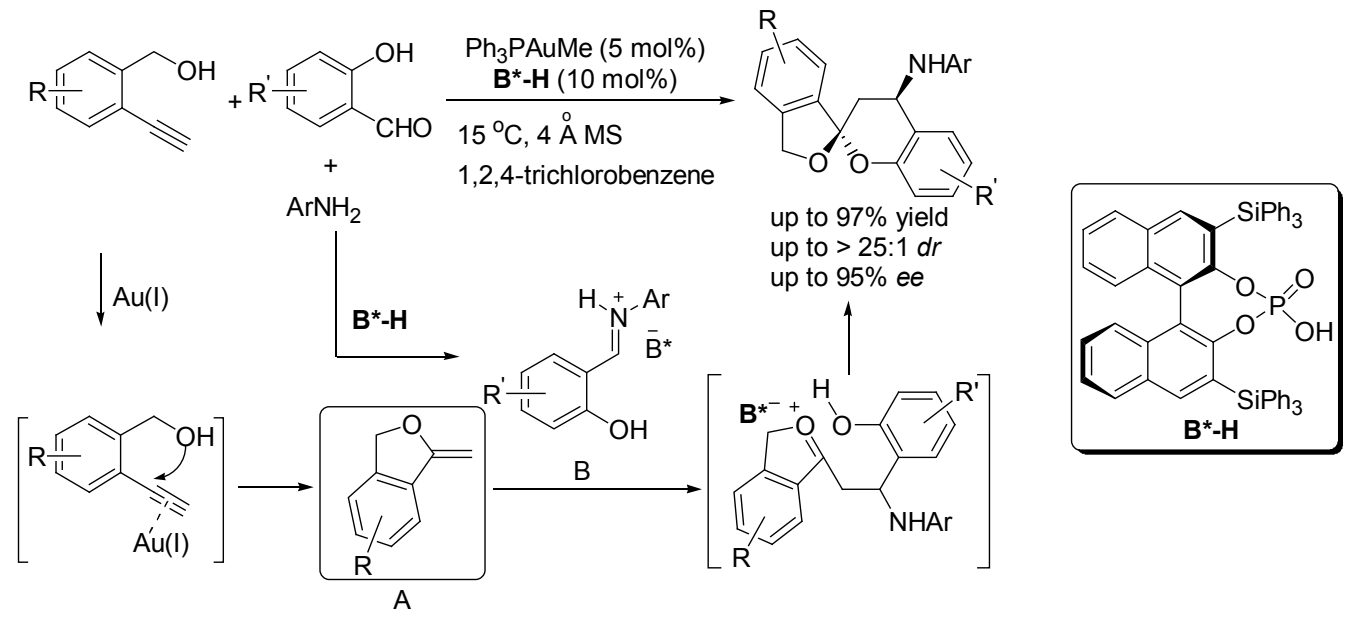

图 20 炔醇环化/形式上的 $[4+2]$ 环化串联反应

Figure 20 Cyclization of alkynols/formal [4+2] cyclization reaction 
化生成烯醇醚 $\mathbf{A}$, 烯醇醚 $\mathbf{A}$ 与水杨醛和苯胺生成的亚胺 B 经由 Mannich 反应和缩酮螺环化反应, 以 $97 \%$ 的收率 和 $95 \%$ 的 $e e$ 值得到芳香螺环缩酮化合物.

2012 年, 龚流柱课题组 ${ }^{[27]}$ 还报道了在金(I)的催化 作用下炔烃硅醇化合物发生环化反应，原位生成的 1,3硅氧取代二烯进一步在手性磷酰胺的催化下与 1,4-䒬醌 发生不对称 Diels-Alder 反应, 高收率、高选择性地得到 多手性中心的多环化合物(图 21). 该反应中, 手性磷酸 的催化效果不及手性磷酰胺.

龚流柱课题组在上述工作的基础上，最近又报道了 金和手性磷酰胺催化的分子内炔烃硅氧化和 Mukaiyama Aldol 反应的串联反应(图 22) ${ }^{[28]}$. 利用产物 中硅原子, 进一步与硔代苯发生 Hiyama 交叉偶联, 生 成联苯化合物, 从而用于合成木脂素类化合物.

\section{7 总结与展望}

金属配合物和有机小分子组合的共催化体系，可以 实现单一催化剂不易控制的不对称催化反应, 也能完成 单一催化剂不能实现的新反应, 已经成为当前有机化学 的一个新兴热门研究领域. 其中, 对金属配合物和手性 磷酸结合的共催化体系研究最为广泛. 但目前大多数金 属配合物/有机小分子双体系催化的不对称接力反应多 集中于金、铑、钉等过渡金属与手性磷酸的结合, 因此 可以针对反应类型的不同, 发展多种金属和多种有机小 分子参与的催化反应体系; 在发展多种多体系催化反应 的同时, 还应进一步扩大反应类型, 使不对称接力催化 反应能够在天然产物全合成中得到更为广泛的应用. 此 外，金属与有机小分子组成的共催化体系在工业生产中 的应用还较少, 因此开发适合工业化生产的催化体系也 是化学家今后共同的任务.

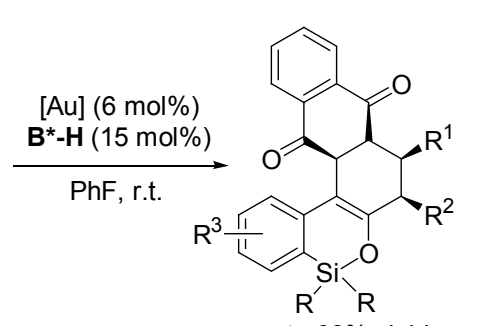

$\downarrow \mathrm{Au}(\mathrm{I})$<smiles>O=C1C=CC(=O)c2ccccc21</smiles>

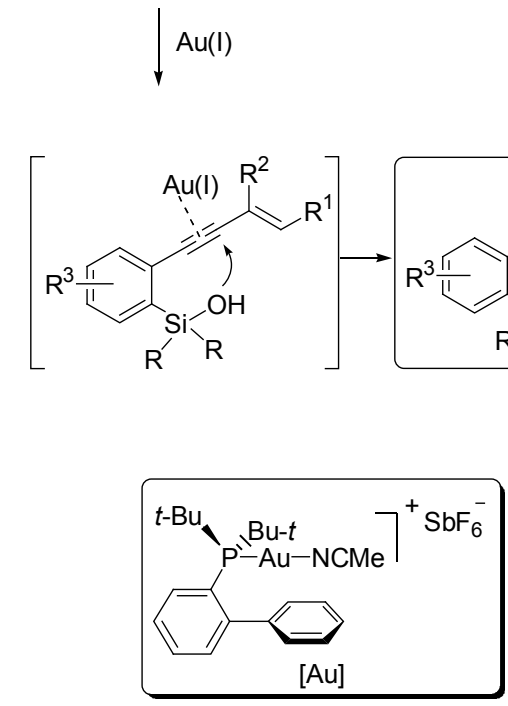

图 21 炔醇环化/Diels-Alder 串联反应

Figure 21 Cyclization of alkynols/Diels-Alder cascade reaction<smiles>[R]C#Cc1c[R1]ccc1[Si](C)(C)O</smiles><smiles>O=C(O[18O])C(O)O[18O]</smiles>

IPrAuMe (5 mol\%) B $^{*}-\mathrm{H}(15 \mathrm{~mol} \%)$

$25^{\circ} \mathrm{C}, 36 \mathrm{~h}$

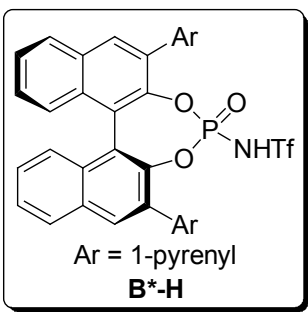

up to $98 \%$ yield

up to $96 \%$ ee<smiles>[R]c1c([R])c2c(c3c1O[Si]([R])([R])c1ccccc1-3)C(=O)c1ccccc1C2=O</smiles>

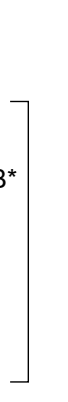




\section{作者简介}

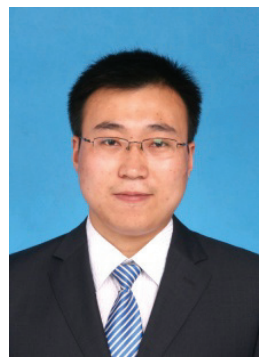

吴祥, 2012 年于南开大学获得博士学位, 同年 8 月进入中 国科学技术大学合肥微尺度物质科学国家实验室从事博士后 研究. 研究方向为不对称接力催化和天然产物全合成.

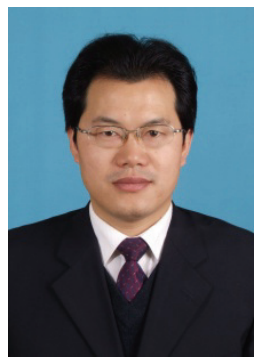

龚流柱, 中国科学技术大学合肥微尺度物质科学国家实 验室(筹)和化学系教授, 博士生导师, 2003 年国家态出青年基 金获得者, 2007 年被评选为教育部 “长江学者奖励计划” 有机 化学学科特聘教授. 主要从事有机合成方法学, 不对称催化和 天然产物合成的研究.

\section{References}

[1] For recent reviews of cooperative catalysis: (a) Shao, Z.; Zhang, H. Chem. Soc. Rev. 2009, 38, 2745; (b) Zhou, J. Chem. Asian J. 2010, 5, 422; (c) Rueping, M.; Koenigs, R. M.; Atodiresei, I. Chem. Eur. J. 2010, 16, 9350; (d) Zhong, C.; Shi, X. Eur. J. Org. Chem. 2010, 16, 2999; (e) Piovesana, S.; Schietroma, D. M. S.; Bella, M. Angew. Chem., Int. Ed. 2011, 50, 6216; (f) Patil, N. T. Angew. Chem., Int. Ed. 2011, 50, 1759; (g) Loh, C. C. J.; Enders, D. Chem.-Eur. J. 2012, 18, 10212; (h) Patil, N. T.; Shinde, V. S.; Gajula, B. Org. Biomol. Chem. 2012, 10, 211; (i) Du, Z.; Shao, Z. Chem. Soc. Rev. 2013, 42, 1337.

[2] Han, Z.-Y.; Wang, C.; Gong, L.-Z. In Science of Synthesis: Asymmetric Organocatalysis, Vol. 2, Ed.: Maruoka, K., Georg Thieme
Verlag, Stuttgart, 2012, p. 697.

[3] (a) Han, Z. Y. Ph.D. Dissertation, University of Science and Technology of China, Hefei, 2011. (韩志勇, 博士论文, 中国科学技术 大学, 合肥, 2011); (b) Wang, C. Ph.D. Dissertation, University of Science and Technology of China, Hefei, 2012. (王超, 博士论文, 中国科学技术大学, 合肥, 2012).

[4] (a) Akiyama, T. Chem. Rev. 2007, 107, 5744; (b) Terada, M. Synthesis 2010, 1929; (c) Yu, J.; Shi, F.; Gong, L.-Z. Acc. Chem. Res. 2011, 44, 1156; (d) Gao, Y.-J.; Yang, L.-H.; Song, S.-J.; Ma, J.-J.; Tang, R.-X.; Bian, R.-H.; Liu, H.-Y.; Wu, Q.-H.; Wang, C. Chin. J. Org. Chem. 2008, 28, 8. (高勇军, 杨丽华, 宋双居, 马晶军, 唐然 肖，边瑞环，刘海燕，吴秋华，王春，有机化学， 2008，28，8); (e) Su, Y.; Shi, F. Chin. J. Org. Chem. 2010, 30, 486. (苏亚军, 史福强, 有机化学, 2010, 30, 486).

[5] Terada, M.; Sorimachi, K. J. Am. Chem. Soc. 2008, 130, 14452.

[6] You, S. L.; Cai, Q.; Zhao, Z. A. Angew. Chem., Int. Ed. 2009, 48, 7428.

[7] Xiao, W. J.; Chen, J. R.; Li, C. F.; An, X. L.; Zhang, J. J.; Zhu, X. Y. Angew. Chem., Int. Ed. 2008, 47, 2489.

[8] Cai, Q.; Zheng, C.; You, S. L. Angew. Chem., Int. Ed. 2010, 49, 8666.

[9] Cai, Q.; Liang, X.-W.; Wang, S.-G.; Zhang, J.-W. Zhang, X.; You, S.-L. Org. Lett. 2012, 14, 5022.

[10] Chen, Q. A.; Wang, D. S.; Zhou, Y. G.; Duan, Y.; Fan, H. J.; Yang, Y.; Zhang, Z. J. Am. Chem. Soc. 2011, 133, 6126.

[11] Chen, Q. A.; Chen, M. W.; Yu, C. B.; Shi, L.; Wang, D. S.; Yang, Y.; Zhou, Y. G. J. Am. Chem. Soc. 2011, 133, 16432.

[12] Chen, Q. A.; Gao, K.; Duan, Y.; Ye, Z. S.; Shi, L.; Yang, Y.; Zhou, Y. G. J. Am. Chem. Soc. 2012, 134, 2442.

[13] Terada, M.; Toda, Y. Angew. Chem., Int. Ed. 2012, 51, 2093.

[14] Terada, M.; Toda, Y.J. Am. Chem. Soc. 2009, 131, 6354

[15] Ren, L.; Lei, T.; Ye, J.-X.; Gong, L.-Z. Angew. Chem., Int. Ed. 2012, 51,771 .

[16] (a) Hashimi, A. S. K. Chem. Rev. 2007, 107, 3180; (b) Li, Z.; Brouwer, C.; He, C. Chem. Rev. 2008, 108, 3239.

[17] Mizushima, E.; Hayashi, T.; Tanaka, M. Org. Lett. 2003, 5, 3349.

[18] Han, Z.-Y.; Xiao, H.; Chen, X.-H.; Gong, L.-Z. J. Am. Chem. Soc. 2009, 131, 9182.

[19] Liu, X.-Y.; Che, C.-M. Org. Lett. 2009, 11, 4204.

[20] Wang, C.; Han, Z.-Y.; Luo, H.; Gong, L.-Z. Org. Lett. 2010, 12, 2266.

[21] Wu, H.; He, Y.-P.; Gong, L.-Z. Adv. Synth. Catal. 2012, 354, 975.

[22] Patil, N. T.; Mutyala, A. K.; Konala, A.; Tella, R. B. Chem. Commun. 2012, 48, 3094.

[23] Patil, N. T.; Raut, V. S.; Tella, R. B. Chem. Commun. 2013, 49, 570.

[24] Muratore, M. E.; Holloway, C. A.; Pilling, A. W.; Storer, R. I.; Trevitt, G.; Dixon, D. J. J. Am. Chem. Soc. 2009, 131, 10796.

[25] Han, Z.-Y.; Guo, R.; Wang, P.-S.; Chen, D.-F.; Xiao, H.; Gong, L.-Z. Tetrahedron Lett. 2011, 52, 5963.

[26] Wu, H.; He, Y.-P.; Gong, L.-Z. Org. Lett. 2013, 15, 460.

[27] Han, Z.-Y.; Chen, D.-F.; Wang, Y.-Y.; Guo, R.; Wang, P.-S.; Wang, C.; Gong, L.-Z. J. Am. Chem. Soc. 2012, 134, 6532.

[28] Wang, P.-S.; Li, K.-N.; Zhou, X.-L.; Wu, X.; Han, Z.-Y.; Guo, R.; Gong, L.-Z. Chem.-Eur. J. 2013, 19, 6234.

(Cheng, F.) 